\title{
Adjuvant mitotane therapy is beneficial in non-metastatic adrenocortical carcinoma at high risk of recurrence
}

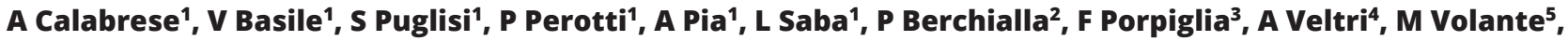 \\ G Reimondo ${ }^{1}$, A Berruti ${ }^{6}$ and $\mathbf{M}$ Terzolo $^{1}$
}

${ }^{1}$ Internal Medicine, Department of Clinical and Biological Sciences, S. Luigi Gonzaga Hospital, University of Turin, Orbassano, Turin, Italy, ${ }^{2}$ Statistical Unit, Department of Clinical and Biological Sciences, University of Turin, Orbassano, Turin, Italy, ${ }^{3}$ Urology, ${ }^{4}$ Radiology, ${ }^{5}$ Pathology, Department of Oncology, S. Luigi Gonzaga Hospital, University of Turin, Orbassano, Turin, Italy, and ${ }^{6}$ Oncology, Department of Medical and Surgical Specialties, Radiological Sciences, and Public Health Medical, ASST-Spedali Civili, University of Brescia, Brescia, Italy

\author{
Correspondence \\ should be addressed \\ to S Puglisi \\ Email \\ sorayapuglisi@yahoo.it
}

\section{Abstract}

Objective: Many patients with adrenocortical carcinoma (ACC) suffer from tumor recurrence despite radical surgery. Evidence on the post-operative use of mitotane is controversial and no predictors of response are available. We aimed to assess whether adjuvant mitotane treatment may prolong survival in patients with non-metastatic ACC following complete resection and whether ACC patients at high risk of recurrence may benefit from treatment.

Design and methods: We retrospectively reviewed data from 152 non-metastatic ACC patients followed at the San Luigi Gonzaga Hospital: 100 patients were treated with adjuvant mitotane and 52 patients were left untreated following surgery. We assessed a number of potential predictive factors of recurrence and death. Mitotane effect was explored stratifying patients by staging (stage I-II vs stage III), hormone secretion (yes vs no) and Ki67 index. Results: The non-treated group had a higher risk of recurrence (HR: $2.79,95 \% \mathrm{Cl}: 1.58-4.91 ; P<0.001$ ) than mitotanetreated group, while overall survival was not significantly different between groups. Hormone secretion, elevated Weiss score and elevated Ki67 index confer a higher risk of both recurrence and death and stage III ACC of death. Adjuvant mitotane treatment reduced significantly the risk of death in patients with elevated Ki67 index $(P=0.005)$ and in patients with stage III ACC $(P=0.02)$.

Conclusions: Adjuvant mitotane may prolong recurrence-free survival in radically resected ACC patients with acceptable toxicity and may also prolong overall survival in a subgroup of ACC patients at high risk of recurrence.

\section{Introduction}

Adrenocortical carcinoma (ACC) is a rare endocrine neoplasia, with a reported incidence of about two cases per million population per year, which affects more frequently the female sex and has a peak incidence in the middle age (1). ACC is characterized by an overall dismal prognosis and its clinical manifestations are either the consequence of steroid excess or mass effect; however, ACC is increasingly found as an adrenal incidentaloma (2).
Surgery is the cornerstone of ACC management and the most effective treatment, while survival is poor when surgical removal of ACC is unfeasible (3). Although radical surgery can be potentially curative, many ACC patients will suffer tumor recurrence following apparently curative resection. Prevention of tumor recurrence is of utmost importance because recurrence impairs significantly life expectancy and quality of life of affected patients $(1,4)$.
Published by Bioscientifica Ltd. 
Therefore, adjuvant concepts look sound and the postoperative use of mitotane as an adjuvant therapy following ACC removal has been the most followed approach (5).

Reported outcomes of adjuvant mitotane therapy have been conflicting between studies and, lacking data from randomized controlled trials, evidence is based only on retrospective studies $(6,7)$ which were often underpowered and did not always include a concomitant non-treated group of untreated patients (5). Previous research from our group showed that adjuvant mitotane was associated with longer recurrence-free survival (RFS) in treated patients with radically resected ACC compared to untreated patients. In that study, confounding by indication was reduced comparing two management strategies applied in different settings and treatment was given according to the center attitude toward adjuvant mitotane (i.e. all patients of a given center were treated or not treated) (8). In 2017, we updated the results of our earlier study after nine additional years of follow-up and confirmed that adjuvant mitotane was associated with prolonged RFS (9). Controversy on adjuvant mitotane still lives, however, as demonstrated by a study published in 2016 showing that adjuvant mitotane therapy was associated with decreased RFS (10). Moreover, the available literature provides scanty information on how treatment was delivered (dosing regimen, duration of treatment, circulating levels, etc.) and none of the studies could identify whether outcome of patients at high risk of recurrence is improved with adjuvant mitotane treatment. This information is key considering that adjuvant mitotane is currently recommended in high-risk patients (7). The condition of high risk of recurrence includes stage III, or R1 resection, or Ki67 index $>10 \%$ according to the recent ESE-ENSAT guidelines (7).

To contribute to the debate on post-operative adjuvant mitotane therapy, we reviewed retrospectively our experience at the San Luigi Gonzaga Hospital, a tertiary center for the care of ACC patients in Italy. The main aims of the study were to assess whether adjuvant mitotane treatment may prolong survival in patients with non-metastatic ACC following complete resection and whether ACC patients at high risk of recurrence may benefit from treatment.

\section{Subjects and methods}

At the San Luigi Gonzaga Hospital, we established in 2001 the Adrenocortical Carcinoma Database with the development of a structured data form to collect comprehensive information of ACC patients managed at our center. Data were obtained from patient interviews and available medical documentation and were processed by skilled and experienced personnel. In case of missing information, further data were actively requested to medical institutions where patients have been previously managed. For the purpose of this study, we retrieved data of patients who underwent radical surgery from July 2001 to July 2015. Due to the referral pattern of our center, most patients have been operated on at other institutions (7\% of patients had surgery in our center) and were referred to us after a histologic diagnosis of ACC was secured for considering adjuvant medical therapy. Follow-up for this study was closed in May 2017. The study was approved by the Ethical Committee of San Luigi Gonzaga Hospital and all patients provided written informed consent.

Inclusion criteria of the study were age $\geq 16$ years; pathologically confirmed diagnosis of ACC according to Weiss score (11) (81\% of pathological diagnoses were reviewed at our center); ENSAT stage I-III at diagnosis; complete macroscopic resection, defined as R0, R1 or RX resection on the basis of surgical and pathologic reports; availability of pre-operative and post-operative CT or magnetic resonance imaging (MRI) scans and complete follow-up information. Exclusion criteria were incomplete tumor staging, ENSAT stage IV, history of other previous or concomitant malignancies, R2 resection; recurrence or death before the landmark point of three months; incomplete follow-up information, concomitant adjuvant chemotherapy and radiotherapy or both and patient inclusion in previous studies. None of the present patients has been included in the ADIUVO study (www.epiclin.it/ adiuvo).

Patients' charts were reviewed and the following information was retrieved for the study: patient's age and sex, date of diagnosis, imaging data, ACC stage, clinical presentation including assessment of hormone secretion, type of surgery, pathology report, adjuvant treatment, date and type of recurrence, treatment of recurrence, last follow-up or death. Date of diagnosis was defined as the date of surgery and conversion to open adrenalectomy was considered as open surgery. Completeness of surgery was established by $\mathrm{R}$ status: $\mathrm{R} 0$, free resected margins; $\mathrm{R} 1$, microscopic involvement of resected margins; RX, not determined, and R2, macroscopic invasion of resected margins. Tumor stage was established according to the ENSAT classification (I-II, confined tumor; III, positive lymph nodes or infiltrating neighboring organs/veins without distant 
metastases; IV, distant metastases (12). Biochemical confirmation of hormone excess was requested to categorize an ACC as hormone secreting. Patients were stratified for Ki67 index (Ki67 $\leq 10 \%$ and Ki67 $>10 \%)$. Date of recurrence was defined as the date of radiological evidence of a new lesion. Recurrence was described as 'local' (involving the adrenal region), 'single distant' (one affected organ) or 'multiple distant' (more than one affected organ). If adjuvant mitotane therapy was instituted, we analyzed mitotane levels on treatment, duration of treatment, reasons of treatment discontinuation and severe toxicity. Mitotane levels were attained by the Lysosafe ${ }^{\circledR}$ service since July 2005 while in the preceding years we got data from in-house measurements, as previously described (13). Duration of treatment was calculated from the date of initiation of therapy until ACC recurrence, or discontinuation of treatment, or end of follow-up, whatever occurring first.

From a total of 217 ACC patients on database, 152 patients fulfilled inclusion/exclusion criteria and were retrospectively included in the study (Fig. 1). Of these, 100 patients underwent adjuvant mitotane therapy after initial surgery (mitotane-treated group) and 52 patients were not treated with any adjuvant treatment following surgery (non-treated group). Adjuvant mitotane was recommended following surgery to all ACC patients till 2008. Since then, post-operative adjuvant mitotane was recommended only to the high-risk patients. However, no treatment was chosen for the patients who were not fit to sustain treatment or did not consent to treatment. The non-treated patients underwent the same surveillance protocol as the mitotane-treated patients. Patient management included follow-up visits, imaging, hormone assessment, routine laboratory tests and measurement of mitotane level for treated patients, every 3-4 months. Mitotane was given according to a low-dose monitored regimen, as previously detailed (14).

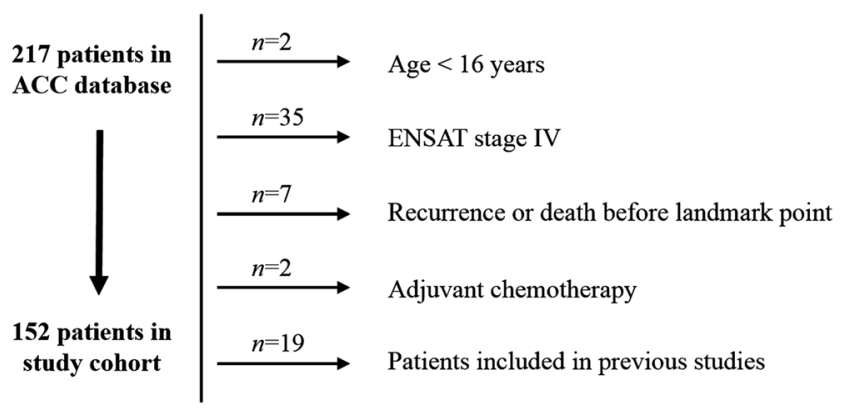

\section{Figure 1}

Study cohort.
Briefly, treatment is started at $1 \mathrm{~g}$ daily with further dose increase every 4-7 days up to 4-6 g daily or the maximum tolerated dose. Mitotane dose is adjusted to patient tolerability and drug levels that are regularly monitored aiming at plasma concentration of $14-20 \mathrm{mg} / \mathrm{L}$. Severe mitotane-related effects leading to hospitalization or treatment discontinuation, either more than 3 months or permanent, were analyzed for the study.

\section{Outcomes}

The main aims of our study were to compare RFS and overall survival (OS) in patients who received adjuvant mitotane therapy after complete macroscopic resection with that of patients who did not receive adjuvant therapy and to identify whether outcome of high-risk patients is improved with treatment. RFS was calculated from the time of initial surgery to the first radiological evidence of recurrence. OS was calculated from the date of initial surgery to the date of death. The following potential predictive factors for either RFS or OS were investigated: patient sex and age, tumor stage, hormone secretion, cortisol excess, type of surgery, R status, Weiss score, Ki67 index, adjuvant mitotane and plasma mitotane levels. Mitotane effect was explored stratifying patients by staging (stage I-II vs stage III), hormone secretion (yes vs no) and Ki67 index.

\section{Statistical analysis}

Categorical data are presented as counts and percentages. Continuous data are presented as medians and ranges. Differences in categorical variables were analyzed by means of the chi-square test, while differences in continuous variables by the two-tailed Mann-Whitney $U$ test. The survival distribution was assessed by the Kaplan-Meier product-limit methods and survival curves were compared by the log-rank test. Patients who did not experience the event (recurrence or death) were censored at the date of the last follow-up visit for the specific survival analysis. Cox proportional hazards regression models were fitted to determine predictive factors on RFS and OS. Proportional hazard assumption was verified by Schoenfeld's residuals and by the log minus log method. To reduce the inherent bias of patients with early progression or death, all survival analyses were performed with the landmark method. Patients who experienced the event (recurrence or death) before the landmark point at 3 months from surgery were excluded from analyses. The time point at 3 months 
was chosen because of the general recommendation that adjuvant mitotane treatment should be initiated within this period (7). In our cohort, $90 \%$ of the patients started mitotane within 3 months, and 100\% were on treatment at 10 months after surgery. Thus, we also did a sensitivity analysis with a time point set at 10 months. Complete case analysis was used. All reported $P$ values are two-sided. $P$ values of less than 0.05 were considered as statistically significant. Statistical analyses were done with $\mathrm{R}$ version 3.4.3.

\section{Results}

\section{Patients}

Baseline characteristics of patients are reported in Table 1. Overall, female sex was mostly affected (61.8\%) and median age at diagnosis was 46 years (range, 16-77). ACC was stage I-II in $75.7 \%$ and hormone secreting in $47.4 \%$ of cases; cortisol was the most frequently secreted hormone, alone or in combination with other steroid hormones. Secreting ACCs were mostly found in women $(P=0.022)$, in younger patients $(P=0.014)$ and had higher Ki67 index $(P=0.025)$, respectively (Supplementary
Table 1, see section on supplementary data given at the end of this article). Surgery was done as an open approach in $71.6 \%$ of cases; $63.5 \%$ in stage I-II and $94.6 \%$ in stage III ACC. R0 resection was attained in $74.2 \%$ of open surgery vs $76.9 \%$ of laparoscopy. Median Weiss score was of 6 (3-9) with a median Ki67 of 20\% (1-70\%); 67.2\% of ACC had Ki67 >10\%. The median follow-up was 51 months, 57 in the mitotane-treated group vs 45 months in the non-treated group. The treated and non-treated groups were evenly distributed according to patient sex, ACC stage, hormone secretion, type of surgery and $\mathrm{R}$ status. The patients of the mitotane-treated group were younger and showed higher Weiss score and Ki67 index than the non-treated group (Table 1).

Among mitotane-treated patients, $47 \%$ of them began adjuvant treatment within 1 month after surgery, $44 \%$ within 3 months and the remaining 9\% between 4 and 10 months. In $62.8 \%$ of patients the therapeutic range of mitotane concentrations (14-20 mg/L) was maintained in at least $50 \%$ of measurements. The patients who maintained the therapeutic range did not show a significant reduction in the risk of recurrence (HR 0.79, (0.41-1.50), $P=0.47)$. Median duration of treatment was 21 months (14 months in patients who

Table 1 Baseline features of patients.

\begin{tabular}{l}
\hline Characteristics \\
\hline Sex, $n$ (\%) \\
Male \\
Female \\
Age at diagnosis, years (median (range)) \\
Tumor stage, $n$ (\%) \\
Stage I-II \\
Stage III \\
Hormone secretion, $n$ (\%) \\
Non-secreting tumor \\
Cortisol \\
Cortisol + other steroids \\
Androgens \\
Aldosterone \\
Other hormones \\
No hormone work up \\
Type of surgery, $n$ (\%) \\
Open adrenalectomy \\
Laparoscopic adrenalectomy \\
R status, $n$ (\%) \\
R0 \\
RX \\
R1 \\
Weiss score, median (range) \\
Ki67 index, \% (median (range)) \\
Ki67 index, $n$ (\%) \\
$\leq 10 \%$ \\
$>10 \%$ \\
\end{tabular}

\begin{tabular}{|c|c|c|}
\hline Mitotane-treated $(n=100)$ & Non-treated $(n=52)$ & $P$ value \\
\hline & & 0.817 \\
\hline $37(37.0)$ & $21(40.4)$ & \\
\hline $63(63.0)$ & 31 (59.6) & \\
\hline \multirow{2}{*}{$45.0(16-77)$} & $49.5(18-70)$ & 0.015 \\
\hline & & 0.580 \\
\hline $77(77.0)$ & $38(73.1)$ & \\
\hline \multirow[t]{2}{*}{$23(23.0)$} & $14(26.9)$ & \\
\hline & & 0.154 \\
\hline $41(41.0)$ & $28(53.8)$ & \\
\hline $23(23.0)$ & $6(11.5)$ & \\
\hline $12(12.0)$ & $3(5.8)$ & \\
\hline $5(5.0)$ & $5(9.6)$ & \\
\hline $1(1.0)$ & $3(5.8)$ & \\
\hline $11(11.0)$ & $3(5.8)$ & \\
\hline \multirow[t]{2}{*}{7 (7.0) } & $4(7.7)$ & \\
\hline & & 0.467 \\
\hline $67(72.0)$ & $34(70.8)$ & \\
\hline \multirow[t]{2}{*}{$26(28.0)$} & $14(29.2)$ & \\
\hline & & 0.435 \\
\hline $71(74.0)$ & 40 (76.9) & \\
\hline $22(22.9)$ & $12(23.1)$ & \\
\hline \multicolumn{3}{|l|}{$3(3.1)$} \\
\hline $6(3-9)$ & $5(3-9)$ & 0.014 \\
\hline \multirow[t]{2}{*}{$20(1-70)$} & $11(1-70)$ & 0.001 \\
\hline & & 0.012 \\
\hline $23(25.3)$ & $21(48.8)$ & \\
\hline $68(74.7)$ & $22(51.2)$ & \\
\hline
\end{tabular}

https://eje.bioscientifica.com 
Table 2 Adverse events of mitotane therapy.

\begin{tabular}{ll}
\hline Type of toxicity & $\boldsymbol{n}(\%)$ \\
\hline Gastrointestinal & $6(37.5)$ \\
Hepatic & $3(18.8)$ \\
Hematological & $1(6.3)$ \\
Neurological & $3(18.7)$ \\
Other & $3(18.7)$ \\
\hline
\end{tabular}

developed recurrence vs 45 months in patients without recurrence). Causes of treatment discontinuation were toxicity $(n=11)$, poor compliance $(n=7)$ or concomitant diseases $(n=4)$. Unwanted effects of mitotane therapy, mainly affected the gastrointestinal system, are reported in Table 2.

\section{Outcomes}

Recurrence occurred in $62.5 \%$ of cases: $56.5 \%$ of stage I-II and $81.1 \%$ of stage III ACC. The median RFS of the overall cohort was 25 months (4-199). Recurrence was mostly observed within 5 years after surgery (93.5\%). Only six patients recurred after 5 years; they had stage II ACC with a Ki67 $\leq 10 \%$ in three cases and no secretion in four of them. The 1-year RFS was $81.2 \%$ and 5-year RFS $38.1 \%$. Recurrence occurred in 55 patients of the mitotane-treated group (55\%) and in 40 of the non-treated group (76.9\%). The median RFS was 36.5 months (4-199) in the mitotane-treated group and 21 months (4-180) in the non-treated group. The 1-year RFS and 5-year RFS were 85 and $44.4 \%$, respectively, in the mitotane-treated group vs 73.6 and $25.1 \%$, respectively, in the non-treated group. RFS of the two groups were significantly different when compared

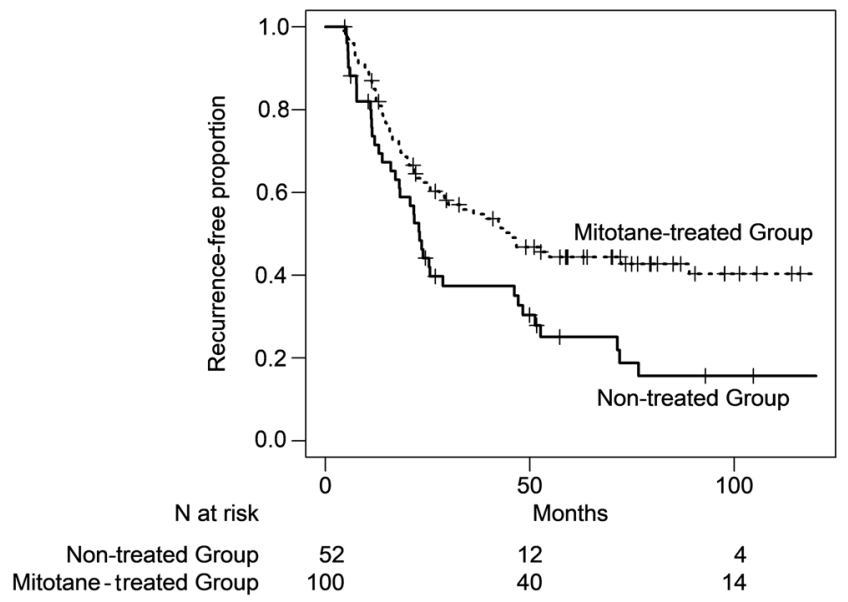

\section{Figure 2}

Kaplan-Meier estimates of recurrence-free survival. with Kaplan-Meier survival analysis $(P<0.001)$ (Fig. 2). A separate analysis with the landmark time point set at ten months confirmed the difference in RFS between the two groups $(P=0.012)$ (Supplementary Fig. 1). After excluding the nine patients who initiated adjuvant mitotane 4-10 months following surgery, univariate analysis showed that stage, hormone secretion, Weiss score, Ki67 index and adjuvant mitotane treatment were independent predictors of recurrence. Multivariate analysis confirmed hormone secretion, Weiss score, Ki67 index and adjuvant mitotane treatment as predictive factors of recurrence (Table 3). After adjusting for prognostic factors, the non-treated group had a higher risk of recurrence (HR 2.79, 95\% CI 1.58-4.91; $P<0.001$ ) than the mitotane-treated group (Fig. 3).

Local recurrence occurred in $20 \%$ of cases, single distant recurrence in $42.2 \%$ and multiple distant recurrences in $37.8 \%$. Loco-regional treatments, including surgery or radiofrequency ablation, were used in $57.6 \%$ of ACC recurrence, while $31.5 \%$ underwent systemic therapy, such as mitotane alone $(17.2 \%)$ or in combination with chemotherapy $(82.8 \%)$. Two patients did not receive any treatment, six patients received multiple treatments and two additional patients received other types of treatment.

Death occurred in $42.1 \%$ of cases; $33 \%$ of stage I-II and $70.3 \%$ of stage III ACC. The median OS of the overall cohort was 57 months (4-231). The 1-year OS was $97.3 \%$ and 5-year OS $64.7 \%$. Death occurred in $40 \%$ patients of the mitotane-treated group and in $46.2 \%$ of the nontreated group. The median OS was 57.5 months (8-199) in the mitotane-treated group and 50.5 months (4-231) in the non-treated group. The 1-year OS and 5-year OS were 99 and $65.7 \%$, respectively, in the mitotane-treated group vs 93.8 and $62.9 \%$, respectively, in the non-treated group. Kaplan-Meier analysis did not find a significant difference between groups $(P=0.85)$ (Fig. 4). A separate analysis with the landmark time point set at 10 months confirmed the absence of a significant difference in OS between the two groups $(P=0.60)$ (Supplementary Fig. 2 ). After excluding the nine patients who initiated adjuvant mitotane 4-10 months following surgery, univariate analysis showed that stage, hormone secretion, Weiss score and Ki67 index were independent predictors of OS. Multivariate analysis confirmed stage, hormone secretion, Weiss score and Ki67 index as predictive factors of OS (Table 3). After adjusting for prognostic factors, OS was not different between the non-treated and the mitotane-treated group (HR 1.22, 95\% CI 0.612.42; $P=0.57$ ) (Fig. 5). 
Table 3 Predictive factors of recurrence-free survival (RFS) and overall survival (OS) in 91 mitotane-treated and 52 non-treated patients.

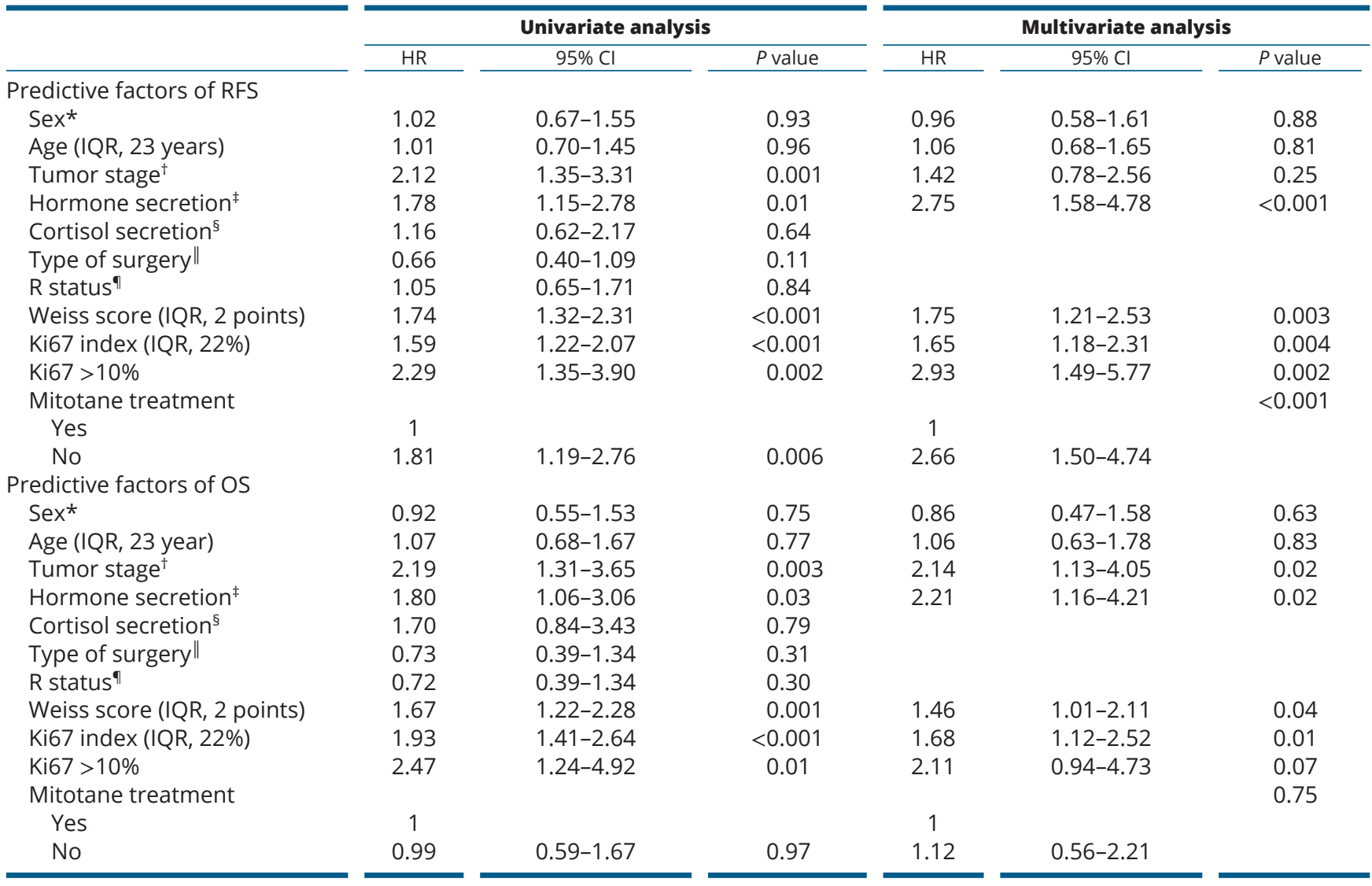

Reference for continuous variables: HR was calculated comparing the interquartile difference (75th percentile minus 25th percentile, IQR). For Weiss score and Ki67 index, a difference in the variable equal to the IQR is associated with a higher risk of recurrence and death. Reference categories: *Male sex, ${ }^{\dagger} \mathrm{ACC}$ stage III, ${ }^{\ddagger}$ Secreting tumors, ${ }^{5}$ Cortisol secretion, "Laparoscopic surgery, "RX-R1.

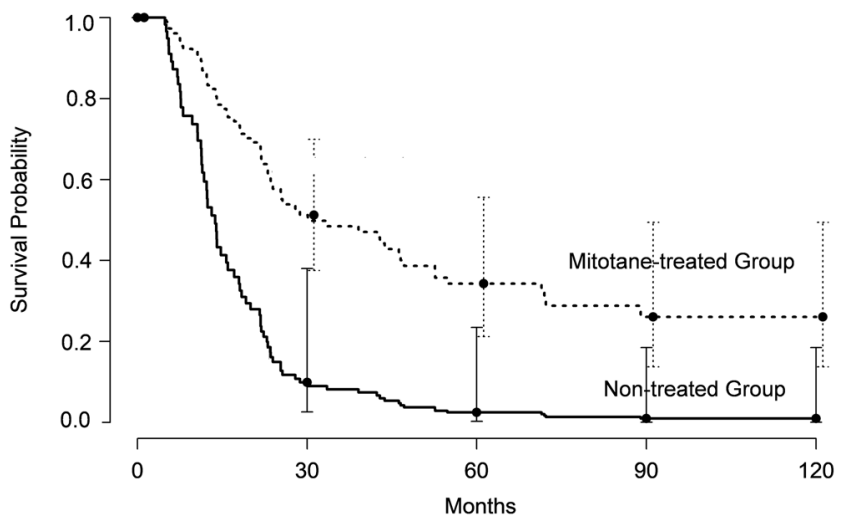

Figure 3

Recurrence-free survival curves adjusted for prognostic factors.

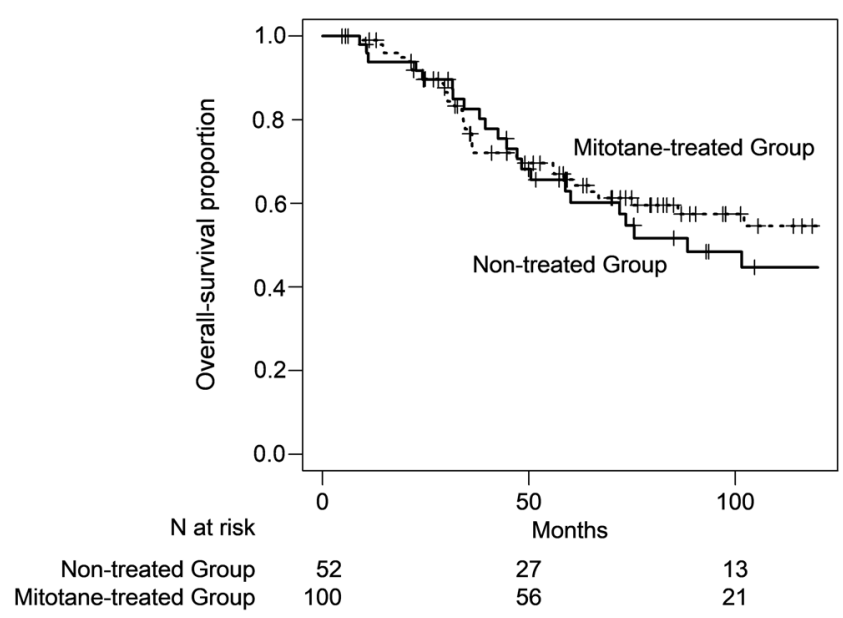

Figure 4

Kaplan-Meier estimates of overall survival. 


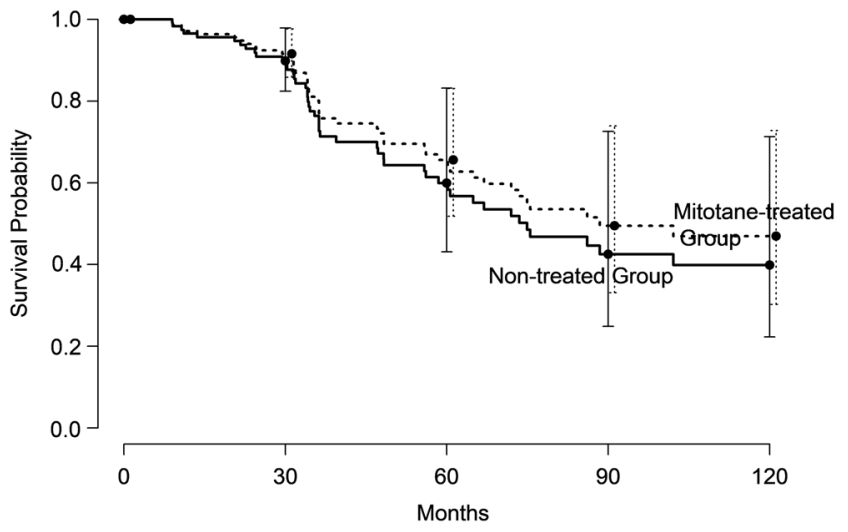

Figure 5

Overall survival curves adjusted for prognostic factors.

Mitotane treatment reduced significantly the risk of both recurrence and death in patients with elevated Ki67 index and the risk of death in patients with stage III ACC. Mitotane treatment was not associated with any difference in survival between patients with secreting or non-secreting ACC (Table 4).

\section{Discussion}

The rarity of ACC has hampered the implementation of large studies, so that only two randomized controlled trials on treatment of advanced ACC are available up to now $(15,16)$ and no randomized study has been conducted in the adjuvant setting. Therefore, evidence on the efficacy of adjuvant mitotane treatment relies on six retrospective studies that included a concomitant group of untreated patients to allow a comparative assessment of treatment efficacy $(9,10,17,18,19,20)$. The multicenter nature of some studies $(9,10,20)$ carries the disadvantage of an inherent heterogeneity in the management of ACC patients and in the modalities of delivering mitotane therapy (i.e. dosing, duration of treatment). Most importantly, no information on the key issue of what characteristics are associated with benefit from adjuvant mitotane treatment can be retrieved from these studies.

In the present study, we report on our experience with adjuvant mitotane treatment at the San Luigi Gonzaga Hospital, a tertiary center for ACC patients in Italy. None of the patients has been included in previous studies. The major findings of the present study are the observation that adjuvant mitotane may prolong RFS and the identification of subgroups of patients that may particularly benefit from treatment. Moreover, we defined predictive factors for recurrence and death.

As expected, patients treated with adjuvant mitotane had worse prognostic factors (higher Weiss score and Ki67 index); however, we observed a significant increase in the risk of recurrence in the untreated patients, whereas the risk of death was similar between untreated and treated patients. That adjuvant mitotane may increase significantly RFS but not OS has been already reported ( 9 , 18) and an explanation for this apparent discrepancy may be an insufficient number of events to reach statistical levels of significance. In our study, however, adjuvant mitotane was associated with a significant increase in OS in patients with elevated Ki67 index or stage III ACC, characteristics associated with a high risk of recurrence. A recent meta-analysis of the available studies demonstrated that adjuvant mitotane was linked with significantly better OS despite some heterogeneity between studies attributable to the variable inclusion criteria but was not able to identify predictors of response (7).

To the best of our knowledge, this is the first evidence supporting the concept that high-risk patients should be treated with adjuvant mitotane, as recommended in previous guidelines on the basis of expert opinion $(6,7)$. In the present study, we were able to identify factors that portend a worse prognosis. Hormone secretion, elevated Weiss score and Ki67 index confer a higher risk of recurrence and death, while stage III predicts shorter OS. ACC stage is generally regarded as the most important prognostic

Table 4 Interaction analysis between mitotane treatment and stage III, hormone secretion and Ki67 index. The table reports the reduction in the relative risk (HR) of recurrence (RFS) and death (OS) associated with the variable of interest in mitotane-treated patients compared with non-treated patients.

\begin{tabular}{|c|c|c|c|c|c|c|}
\hline \multirow[b]{2}{*}{ ACC characteristics } & \multicolumn{3}{|c|}{ RFS } & \multicolumn{3}{|c|}{ os } \\
\hline & $\mathrm{HR}$ & $95 \% \mathrm{Cl}$ & $P$ value & $\mathrm{HR}$ & $95 \% \mathrm{Cl}$ & $P$ value \\
\hline Stage III & 0.96 & $0.32-2.92$ & 0.95 & 0.21 & $0.06-0.78$ & 0.02 \\
\hline Hormone secretion & 0.58 & $0.21-1.62$ & 0.30 & 2.53 & $0.69-9.37$ & 0.16 \\
\hline Ki67 index (1\% increase) & 0.97 & $0.94-0.99$ & 0.02 & 0.94 & $0.90-0.98$ & 0.005 \\
\hline
\end{tabular}


factor $(4,7,12,21,22)$ and was a strong predictor of death also in our cohort. Evidence that the capability of ACC to secrete hormone is a negative prognostic factor is growing $(18,23,24)$ and we confirmed and extended this finding showing that secreting tumors may be more aggressive due to a higher proliferation rate, since they had an elevated Ki67 index compared to non-secreting ACC. This finding is novel and fits well with the observation that hormone secretion is linked to a transcriptome signature typical of aggressive ACC (25). We found that secreting ACCs were more frequent in younger patients and in women, while hormone secretion did not affect efficacy of mitotane treatment, a controversial issue in previous studies $(9,19)$. The Weiss score has not been uniformly considered as a predictor of long-term outcome (26) while higher Ki67 index have been consistently associated with poor outcome $(20,27)$. The present findings support the concept that a $\mathrm{Ki} 67$ index $>10 \%$ is a predictor of high risk for either recurrence or death. This is clinically relevant since a cut-off at $10 \%$ has been proposed to categorize patients for adjuvant treatment (7), a concept currently tested in the ongoing ADIUVO study, the first randomized controlled trial on post-operative adjuvant mitotane in ACC patients (www.epiclin.it/adiuvo).

In our experience, toxicity associated to adjuvant mitotane was acceptable, even if we should acknowledge the fact that unwanted effects might be underestimated in a retrospective analysis. Severe toxicity was recorded in only 16 patients, and 11 of them were not able to tolerate chronic mitotane treatment. Management of patients on adjuvant mitotane is complex, since it implies specific experience to adjust supportive therapy, a careful follow-up to cope with patient needs and regular monitoring of drug levels (14). Mitotane monitoring was likely a key in limiting severe neurological toxicity. Our findings support the view that expert centers should manage such patients and that a low-dose starting regimen may increase tolerability and compliance. Mitotane is generally viewed as a toxic drug (28) but very few data on how treatment was delivered and on related toxicity are available (8). Despite using a low-dose approach, $62.8 \%$ of patients had their mitotane concentrations in the $14-20 \mathrm{mg} / \mathrm{L}$ range for at least $50 \%$ of measurements. In the only study reporting about mitotane levels in an adjuvant setting, $<50 \%$ of patients were at target (10). We were not able to confirm a relationship between the target mitotane concentration and survival advantage (29), and this may suggest that lower levels could be efficacious in an adjuvant setting or that a more precise estimate of chronic mitotane exposure is needed.
Strengths of the present study are the accurate characterization and uniform management of the patients who were cared by the same team of physicians during their disease course. This allowed to capture details of mitotane treatment that were not available in previous studies. We should acknowledge the limits of a retrospective analysis, although we limited bias with landmark analysis and central review of pathological and radiological materials, and the fact that primary surgery was done outside our institution in most patients. However, this pattern of patient referral is typical of tertiary centers $(17,18,30)$.

To conclude, our study shows that adjuvant mitotane treatment prolongs survival in non-metastatic ACC patients and is effective in patients at high risk of recurrence. Use of a low-dose mitotane regimen with careful patient follow-up accomplishes sustained adherence to therapy.

\section{Supplementary data}

This is linked to the online version of the paper at https://doi.org/10.1530/ EJE-18-0923.

\section{Declaration of interest}

$\mathrm{M} T$ has received research grant from HRA Pharma and Novartis and advisory board honoraria from HRA Pharma. A B has received advisory board honoraria from HRA Pharma. S P has received research grant from HRA Pharma. The other authors have nothing to disclose. Partial data of this work were presented at the ENDO 2018 and the 20th European Congress of Endocrinology as posters.

\section{Funding}

This work was supported by a research grant from Associazione Italiana per la Ricerca sul Cancro (grant number IG17678).

\section{Author contribution statement}

$M T$ and $A B$ designed the study, interpreted the results and revised the final version. $V B, S P, A P, G R$ and $M T$ managed the patients. $L S$ did hormone measurements. A C, V B and P P collected data and contributed to data interpretation. $\mathrm{P} P$ took care of ethic commitments. A C and P B did the statistical analyses. F P did the surgical operations. A $V$ did the central radiologic review. $M \vee$ did the central pathological review. A C, V B and $S$ $P$ wrote the first draft of the report. All authors made critical revisions of the report.

\section{References}

1 Kerkhofs TMA, Verhoeven RHA, Van der Zwan JM, Dieleman J, Kerstens MN, Links TP, Van de Poll-Franse LV \& Haak HR. Adrenocortical carcinoma: a population-based study on incidence and survival in the Netherlands since 1993. European Journal of Cancer 201349 2579-2586. (https://doi.org/10.1016/j. ejca.2013.02.034) 
2 Fassnacht M, Arlt W, Bancos I, Dralle H, Newell-Price J, Sahdev A, Tabarin A, Terzolo M, Tsagarakis S \& Dekkers OM. Management of adrenal incidentalomas: European Society of Endocrinology Clinical Practice Guideline in collaboration with the European Network for the study of adrenal tumors. European Journal of Endocrinology 2016 175 G1-G34. (https://doi.org/10.1530/EJE-16-0467)

3 Gaujoux S, Mihai R \& Joint Working Group of ESES and ENSAT. European Society of Endocrine Surgeons (ESES) and European Network for the Study of Adrenal Tumours (ENSAT) recommendations for the surgical management of adrenocortical carcinoma. British Journal of Surgery 2017104 358-376. (https://doi. org/10.1002/bjs.10414)

4 Crucitti F, Bellantone R, Ferrante A, Boscherini M \& Crucitti P. The Italian registry for adrenal cortical carcinoma: analysis of a multiinstitutional series of 129 patients. Surgery 1996119 161-170. (https://doi.org/10.1016/S0039-6060(96)80164-4)

5 Terzolo M \& Berruti A. Adjunctive treatment of adrenocortical carcinoma. Current Opinion in Endocrinology, Diabetes and Obesity 2008 15 221-226. (https://doi.org/10.1097/MED.0b013e3282fdf4c0)

6 Berruti A, Baudin E, Gelderblom H, Haak HR, Porpiglia F, Fassnacht M, Pentheroudakis G \& ESMO Guidelines Working Group. Adrenal cancer: ESMO Clinical Practice Guidelines for diagnosis, treatment and follow-up. Annals of Oncology 201223 vii131-vii138. (https://doi.org/10.1093/annonc/mds231)

7 Fassnacht M, Dekkers OM, Else T, Baudin E, Berruti A, de Krijger RR, Haak HR, Mihai R, Assie G \& Terzolo M. European Society of Endocrinology Clinical Practice Guidelines on the management of adrenocortical carcinoma in adults, in collaboration with the European Network for the Study of Adrenal Tumors. European Journal of Endocrinology 2018179 G1-G46. (https://doi.org/10.1530/EJE-180608)

8 Terzolo M, Angeli A, Fassnacht M, Daffara F, Tauchmanova L, Conton PA, Rossetto R, Buci L, Sperone P, Grossrubatscher E et al. Adjuvant mitotane treatment for adrenocortical carcinoma. New England Journal of Medicine 2007356 2372-2380. (https://doi. org/10.1056/NEJMoa063360)

9 Berruti A, Grisanti S, Pulzer A, Claps M, Daffara F, Loli P, Mannelli M, Boscaro M, Arvat E, Tiberio G et al. Long-term outcomes of adjuvant mitotane therapy in patients with radically resected adrenocortical carcinoma. Journal of Clinical Endocrinology and Metabolism 2017102 1358-1365. (https://doi.org/10.1210/jc.2016-2894)

10 Postlewait LM, Ethun CG, Tran TB, Prescott JD, Pawlik TM, Wang TS, Glenn J, Hatzaras I, Shenoy R, Phay JE et al. Outcomes of adjuvant mitotane after resection of adrenocortical carcinoma: a 13-institution study by the US Adrenocortical Carcinoma Group. Journal of the American College of Surgeons 2016222 480-490. (https://doi. org/10.1016/j.jamcollsurg.2015.12.013)

11 Weiss LM, Medeiros LJ \& Vickery AL. Pathologic features of prognostic significance in adrenocortical carcinoma. American Journal of Surgical Pathology 198913 202-206. (https://doi. org/10.1097/00000478-198903000-00004)

12 Fassnacht M, Johanssen S, Quinkler M, Bucsky P, Willenberg HS, Beuschlein F, Terzolo M, Mueller HH, Hahner S \& Allolio B. Limited prognostic value of the 2004 International Union Against Cancer staging classification for adrenocortical carcinoma. Cancer 2009115 243-250. (https://doi.org/10.1002/cncr.24030)

13 De Francia S, Pirro E, Zappia F, De Martino F, Sprio AE, Daffara F, Terzolo M, Berruti A, Di Carlo F \& Ghezzo F. A new simple HPLC method for measuring mitotane and its two principal metabolites. Journal of Chromatography B 2006837 69-75. (https://doi. org/10.1016/j.jchromb.2006.04.005)

14 Terzolo M, Ardito A, Zaggia B, Laino F, Germano A, De Francia S, Daffara F \& Berruti A. Management of adjuvant mitotane therapy following resection of adrenal cancer. Endocrine 201242 521-525. (https://doi.org/10.1007/s12020-012-9719-7)
15 Fassnacht M, Terzolo M, Allolio B, Baudin E, Haak H, Berruti A, Welin S, Schade-Brittinger C, Lacroix A, Jarzab B et al. Combination chemotherapy in advanced adrenocortical carcinoma. New England Journal of Medicine 2012366 2189-2197. (https://doi.org/10.1056/ NEJMoa1200966)

16 Fassnacht M, Berruti A, Baudin E, Demeure MJ, Gilbert J, Haak H, Kroiss M, Quinn DI, Hesseltine E, Ronchi CL et al. Linsitinib (OSI906) versus placebo for patients with locally advanced or metastatic adrenocortical carcinoma: a double-blind, randomised, phase 3 study. Lancet Oncology 201516 426-435. (https://doi.org/10.1016/ S1470-2045(15)70081-1)

17 Grubbs EG, Callender GG, Xing Y, Perrier ND, Evans DB, Phan AT $\&$ Lee JE. Recurrence of adrenal cortical carcinoma following resection: surgery alone can achieve results equal to surgery plus mitotane. Annals of Surgical Oncology 201017 263-270. (https://doi. org/10.1245/s10434-009-0716-x)

18 Else T, Williams AR, Sabolch A, Jolly S, Miller BS \& Hammer GD. Adjuvant therapies and patient and tumor characteristics associated with survival of adult patients with adrenocortical carcinoma. Journal of Clinical Endocrinology and Metabolism 201499 455-461. (https:// doi.org/10.1210/jc.2013-2856)

19 Bertherat J, Coste J \& Bertagna X. Adjuvant mitotane in adrenocortical carcinoma (letter to the editor). New England Journal of Medicine 2007356 2372-2380.

20 Beuschlein F, Weigel J, Saeger W, Kroiss M, Wild V, Daffara F, Libé R, Ardito A, Al Ghuzlan A, Quinkler M et al. Major prognostic role of Ki67 in localized adrenocortical carcinoma after complete resection. Journal of Clinical Endocrinology and Metabolism 2015100 841-849. (https://doi.org/10.1210/jc.2014-3182)

21 Kebebew E, Reiff E, Duh QY, Clark OH \& McMillan A. Extent of disease at presentation and outcome for adrenocortical carcinoma: have we made progress? World Journal of Surgery 200630 872-878. (https://doi.org/10.1007/s00268-005-0329-x)

22 Lughezzani G, Sun M, Perrotte P, Jeldres C, Alasker A, Isbarn H, Budäus L, Shariat SF, Guazzoni G, Montorsi F et al. The European Network for the study of adrenal tumors staging system is prognostically superior to the international union against cancer-staging system: a North American validation. European Journal of Cancer 201046 713-719. (https://doi.org/10.1016/j. ejca.2009.12.007)

23 Berruti A, Fassnacht M, Haak H, Else T, Baudin E, Sperone P, Kroiss M, Kerkhofs T, Williams AR, Ardito A et al. Prognostic role of overt hypercortisolism in completely operated patients with adrenocortical cancer. European Urology 201465 832-838. (https://doi.org/10.1016/j. eururo.2013.11.006)

24 Margonis GA, Kim Y, Tran TB, Postlewait LM, Maithel SK, Wang TS, Glenn JA, Hatzaras I, Shenoy R, Phay JE et al. Outcomes after resection of cortisol-secreting adrenocortical carcinoma. American Journal of Surgery 2016211 1106-1113. (https://doi.org/10.1016/j. amjsurg.2015.09.020)

25 Zheng S, Cherniack AD, Dewal N, Moffitt RA, Danilova L, Murray BA, Lerario AM, Else T, Knijnenburg TA, Ciriello G et al. Comprehensive pan-genomic characterization of adrenocortical carcinoma. Cancer Cell 201630 363. (https://doi.org/10.1016/j.ccell.2016.07.013)

26 Volante M, Bollito E, Sperone P, Tavaglione V, Daffara F, Porpiglia F, Terzolo M, Berruti A \& Papotti M. Clinicopathological study of a series of 92 adrenocortical carcinomas: from a proposal of simplified diagnostic algorithm to prognostic stratification. Histopathology 2009 55 535-543. (https://doi.org/10.1111/j.1365-2559.2009.03423.x)

27 Morimoto R, Satoh F, Murakami O, Suzuki T, Abe T, Tanemoto M, Abe M, Uruno A, Ishidoya S, Arai Y et al. Immunohistochemistry of a proliferation marker Ki67/MIB1 in adrenocortical carcinomas: Ki67/ MIB1 labeling index is a predictor for recurrence of adrenocortical carcinomas. Endocrine Journal 200855 49-55. (https://doi. org/10.1507/endocrj.K07-079) 
28 Veytsman I, Nieman L \& Fojo T. Management of endocrine manifestations and the use of mitotane as a chemotherapeutic agent for adrenocortical carcinoma. Journal of Clinical Oncology 200927 4619-4629. (https://doi.org/10.1200/JCO.2008.17.2775)

29 Terzolo M, Baudin AE, Ardito A, Kroiss M, Leboulleux S, Daffara F, Perotti P, Feelders RA, deVries JH, Zaggia B et al. Mitotane levels predict the outcome of patients with adrenocortical carcinoma treated adjuvantly following radical resection. European Journal of
Endocrinology 2013169 263-270. (https://doi.org/10.1530/EJE-130242)

30 Fassnacht M, Johanssen S, Fenske W, Weismann D, Agha A, Beuschlein F, Führer D, Jurowich C, Quinkler M, Petersenn S et al. Improved survival in patients with stage II adrenocortical carcinoma followed up prospectively by specialized centers. Journal of Clinical Endocrinology and Metabolism 201095 4925-4932. (https://doi. org/10.1210/jc.2010-0803)

Received 16 November 2018

Revised version received 6 April 2019

Accepted 16 April 2019 